INPLASY

PROTOCOL

To cite: Ling et al. Accuracy of ultrasound in distinguishing pathology of malignant thyroid diseases: A protocol for systematic review and metaanalysis. Inplasy protocol 2021120072. doi: 10.37766/inplasy2021.12.0072

Received: 16 December 2021

Published: 16 December 2021

Corresponding author: Ling Qin

dlqinling@163.com

Author Affiliation:

Ultrasound department of the First Affiliated Hospital of

Dalian Medical University.

Support: Hui Wang.

Review Stage at time of this submission: Completed but not published.

Conflicts of interest:

None declared.

\section{Accuracy of ultrasound in distinguishing pathology of malignant thyroid diseases: A protocol for systematic review and meta-analysis}

Ling, Q1; Qiyu, L2; Hui, W3; Lipeng, S4.

Review question / Objective: This meta-analysis aimed to determine the accuracy of ultrasound in distinguishing pathology of malignant thyroid diseases.

Eligibility criteria: Type of study. This study will only include high quality clinical cohort or case control studies. Type of patients. The patients should be those who had undergone breast diseases. Intervention and comparison. This study compares Al with pathology for diagnosing breast diseases. Type of outcomes. The primary outcomes include sensitivity, specificity, positive and negative likelihood ratio, diagnostic odds ratio, and the area under the curve of the summary receiver operating characteristic.

INPLASY registration number: This protocol was registered with the International Platform of Registered Systematic Review and Meta-Analysis Protocols (INPLASY) on 16 December 2021 and was last updated on 16 December 2021 (registration number INPLASY2021120072).

\section{INTRODUCTION}

Review question / Objective: This metaanalysis aimed to determine the accuracy of ultrasound in distinguishing pathology of malignant thyroid diseases.

Rationale: Previous studies showed that ultrasound has high effectiveness and reliability in distinguishing pathology of malignant thyroid diseases .However, the results of these studies have been contradictory.

Condition being studied: Accuracy of ultrasound in distinguishing pathology of malignant thyroid diseases. 


\section{METHODS}

Search strategy: The following keywords and MeSH terms were used: [breast cancer or breast tumors or breast diseases ] and [artificial intelligence or Al or Al-assisted diagnosis system]. We also performed a manual search to find other potential articles.

Participant or population: The patients should be those who had undergone breast diseases.

Intervention: This study compares ultrasound with pathology for distinguishing pathology of malignant thyroid diseases.

Comparator: This study compares ultrasound with pathology for distinguishing pathology of malignant thyroid diseases.

Study designs to be included: This study will only include high quality clinical cohort or case control studies.

Eligibility criteria: Type of study. This study will only include high quality clinical cohort or case control studies. Type of patients. The patients should be those who had undergone breast diseases. Intervention and comparison. This study compares Al with pathology for diagnosing breast diseases. Type of outcomes. The primary outcomes include sensitivity, specificity, positive and negative likelihood ratio, diagnostic odds ratio, and the area under the curve of the summary receiver operating characteristic.

Information sources: PubMed, Web of Science, Cochrane Library, and Chinese biomedical databases will be searched from their inceptions to the October 20, 2021, without language restrictions.

Main outcome(s): The primary outcomes include sensitivity, specificity, positive and negative likelihood ratio, diagnostic odds ratio, and the area under the curve of the summary receiver operating characteristic.
Data management: Two authors will independently select the trials according to the inclusion criteria, and import into Endnote X9 (Thomson Corporation, Stanford, USA).

Quality assessment / Risk of bias analysis: Methodological quality was independently assessed by two researchers based on the quality assessment of studies of diagnostic accuracy studies (QUADAS) tool.

Strategy of data synthesis: We will calculate the pooled summary statistics for sensitivity (Sen), specificity (Spe) with their $95 \%$ confidence intervals (CIs). The summary receiver operating characteristic (SROC) curve and corresponding area under the curve (AUC) will be obtained.

Subgroup analysis: The Cochran's Qstatistic and 12 test will be used to evaluate potential heterogeneity between studies. If the Q-test shows a $\mathbf{P} 50 \%$, indicating significant heterogeneity, and the random effect model will be employed or if heterogeneity is not significant, the fixedeffects model was used.

Sensitivity analysis: Sensitivity analysis will be performed to evaluate the influence of a single study on the overall estimate.

Country(ies) involved: China.

Keywords: ultrasound, thyroid cancer, pathology.

Contributions of each author:

Author 1 - Ling Qin.

Email: dlqinling@163.com

Author 2 - Qiyu Liu.

Email: 863271992@qq.com

Author 3 - Hui Wang.

Email: Indlnsd@163.com

Author 4 - Lipeng Sun.

Email: sunlp@163.com 